\title{
A Self-Similar Point Process Through Fractal Construction
}

\author{
Manjunath A. Krishnam, Anand Venkatachalam, and Jeffrey M. Capone \\ Telecommunications Research Center (TRC) \\ Department of Electrical Engineering \\ Arizona State University, Tempe, AZ 85287-7206 \\ \{manjunath, anandv, jcapone\} Casu.edu
}

\begin{abstract}
A self-similar point process is developed by embedding a process with bursty behavior over timescales. This embedding is not arbitrary, but achieved through a model which itself has fractal patterns. This model decomposes the self-similar point process over its timescales in a manner that may be tractable for accurate characterization and control of packet traffic. The limiting behavior of the model is shown to possess the properties of a self-similar point process, namely, bursts of arrivals which have no (intrinsic) timescale. Furthermore, this model leads to efficient synthesis of such a process.
\end{abstract}

\section{Introduction}

In 1,2 the term self-similarity is used to describe the fractal (a form of invariance with respect to changes in scale) nature of a stochastic process in its distribution, more specifically, $Y(t)={ }_{d} c^{-H} Y(c t)$, where $=_{d}$ is equality in distribution. Fractal (or self-similar) processes are among many possible models for generating long memory (long-range) dependent process. For example, (fractional) Brownian motion is a self-similar process and its increments may yield a long-ra nge dependent process. Not every fractal process will give rise to long range dependent process, for example standard Brownian motion and its increments.

Long-range dependence can be observed in many ways. One of the most common ways is to examine the correlation function, $\rho(k)$. A long-range dependent process will have a correlation structure that decays to zero at a rate that is proportional to $k^{-\alpha}$ where $0<\alpha<1$, so that $\sum_{k=n}^{\infty} \rho(k)=\infty$, $\forall n<\infty$. A consequence of this correlation structure is that the variance of the sample mean of the process decays to zero at a rate slower than $n^{-1}$, $\lim _{n}$ rightarrow $\infty \frac{\operatorname{var}\left[\sum_{i=1}^{n} X_{i}\right]}{n^{2-\alpha}}=K$ for $0<\alpha<1$, which has been typically referred to as asymptotic self-similarity [34,56].

Asymptotic self-similar traffic has been observed to occur in many communication networks 3178 . This traffic tends to be so bursty, that even bursts are composed of bursts of bursts (a fractal "like" property). Several useful models have been proposed that capture this behavior, namely the $M|G| \infty$ (with Pareto service times) model [9], the superposition of two state Markovian sources [10], 
the mixture of exponentials to fit the long-tail distributions citeFeldman, the superposition of $N$ On-Off processes with sub-exponential $O n$ periods 1213 , deterministic chaotic maps 14 and self-similar (fractal) point processes [1 154]. In each of the above models, it has been shown that the number of arrivals over an interval (number of busy servers in $M|G| \infty$ model) all exhibit a long-range dependent correlation structure.

In this work, a self-similar (fractal) point process is developed. A self-similar point process, as defined by [1] is one whose inter-arrival times follow the "uniformly self-similar law of probability." Mathematically, $\operatorname{Pr}\left(\frac{U}{\epsilon_{1}}>u \mid \frac{U}{\epsilon_{1}}>1\right)=$ $\operatorname{Pr}\left(\frac{U}{\epsilon_{2}}>u \mid \frac{U}{\epsilon_{2}}>1\right)$, where $U$ is the random variable corresponding to the interarrival times. The model developed in this work, is based on a fractal construction of a basic point process (c luster process), where clusters are embedded over an infinite number of timescales. This construction is the basis of a self-similar point process where points appear to be grouped in clusters, which in-turn are grouped in clusters of clusters, etc. [1. This construction leads to bursts which have no (intrinsic) timescale. In addition, this model decomposes the self-similar point process in a manner that may be tractable for the accurate characterization and control of packet traffic. The model may also allow for further understanding the features of self-similar traffic and how they may impact network performance. Furthermore, this model leads to efficient synthesis of such a process.

\section{Construction of a Self-Similar Point Process}

In this section, a model for self-similar point processes is developed. The model is based on a fractal construction of a basic point (cluster) process, where clusters are embedded over all timescales.

\subsection{Basic Process}

Consider a process that generates Poisson arrivals with rate $\lambda$. After each arrival instant, a decision is made with probability $p$ to continue generating arrivals with rate $\lambda$ or with probability $1-p=q$ to turn off for a period of time. The number of arrivals, $N$, before entering an Off period is geometrically distributed with a mean $q^{-1}$. Thus, the time spent in an $O n$ period, $\tau=\sum_{i=1}^{N} X_{i}$, is a geometric sum of independent identically distributed ( $i i d$ ) exponential random variables, $X_{i}$. It is shown in the Appendix, that $\tau$ is an exponentially distributed random variable with parameter $\lambda q$. Therefore, if the Off periods are taken to be exponentially distributed, then an On-Off Markovian model, as shown in Fig. 1. may be used to describe this basic process. The timescale of this basic point process is $\frac{1}{q}$. A realization of the point process generated by this basic On-Off process is seen in Fig . 2 The exponential parameter of the Off period is also taken to be $\lambda q$. In addition, it is also assumed that an arrival is generated upon departing from an Off period. The inter-arrival time probability density function for this basic process is, $f_{1 X}(x)=f_{1 X \mid \text { on }}(x) P_{\text {on }}+$ 


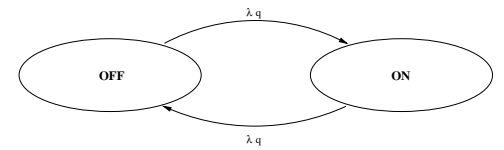

Fig. 1. On-Off Markovian model.

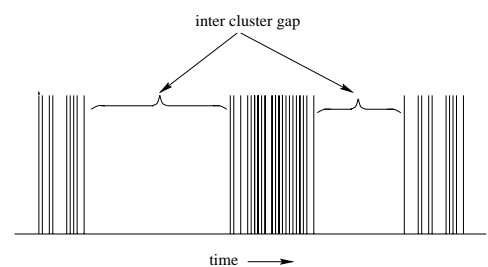

Fig. 2. Point process generated by an On-Off Markovian model.

$f_{1 X \mid \text { off }}(x) P_{\text {off }}$, where $f_{1 X \mid \text { on }}(x)$ and $f_{1 X \mid \text { off }}(x)$ are the inter-arrival distributions while in the $O n$ and $O f f$ states, respectively. $P_{o n}$ is the probability that an inter-arrival time is drawn from the $f_{X \mid \text { on }}(x)$ distribution. Pon may be viewed as, $P_{\text {on }}=\frac{E[\# \text { of arrivals during an on period over one cycle }]}{E[\text { total \# of arrivals over one cycle }]}=\frac{1}{1+q}$. Similarly $P_{o f f}=\frac{q}{1+q}$. The inter-arrival distribution of this process is,

$$
f_{1 X}(x)= \begin{cases}\frac{1}{1+q} \lambda e^{-\lambda x}+\frac{q}{1+q} \lambda q e^{-\lambda q x} & \text { for } x \geq 0 \\ 0 & \text { otherwise. }\end{cases}
$$

This basic model along with its properties are repeatedly used for the fractal construction of a self-similar point process.

\subsection{Fractal Construction}

As it can be seen in Fig. 2] the basic process generates a burst of arrivals over one timescale. The burst lengths are exponentially distributed with parameter $\lambda q$ and the idle periods are also exponentially distributed. To obtain burstiness on the next timescale, this basic process is embedded into the On state of another basic process. This two-scale process may be viewed on the next timescale as the basic process. However, an arrival on this next timescale represents a clus ter on the lower timescale. Furthermore, the inter-cluster gaps, as seen in Fig. 2 are exponentially distributed with parameter $\lambda q$. Therefore, arrivals on this next timescale during an $O n$ period may also be viewed as occurring according to a Poisson process with rate $\lambda q$.

Again, upon each arrival (cluster at the lower timescale), a decision 11 is made with probability $q$ to enter an idle period. Thus, the time spent in the $O n$ state at this next timescale is a geometric sum of iid exponential random variables ( $O n$ and $O f f$ periods of the lower scale) with parameter $\lambda q$, and hence, the arrival On per iod is exponentially distributed with parameter $\lambda q^{2}$. The Off period at this next scale is also taken to be exponentially distributed with parameter $\lambda q^{2}$. When the process described above enters an $O n$ period, the state of the next lower scale process is selected with equal probability. A diagram for this two

${ }^{1}$ A decision is made at the beginning and ending of each arrival to maintain a geometric sum of iid exponential random variables. 

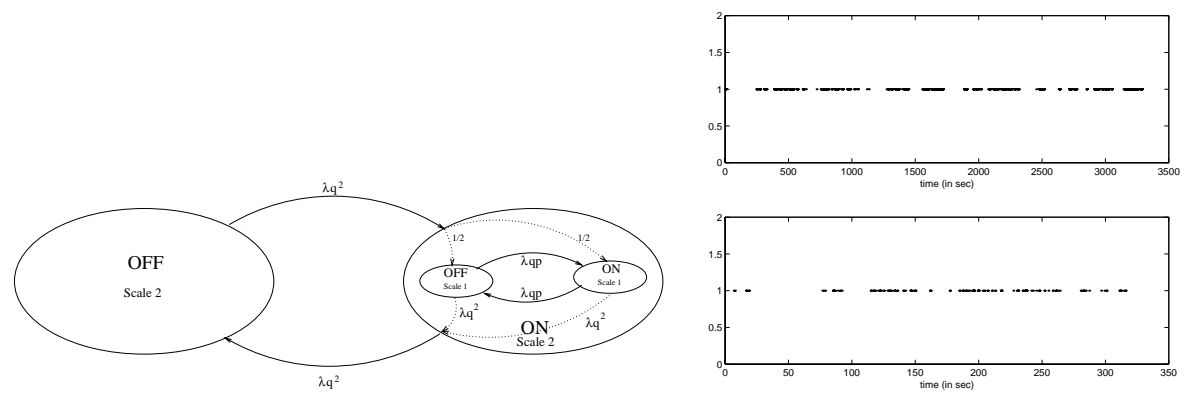

Fig. 3. Embedding an On-Off model of one scale Fig. 4. Point process with one level into another. of embedding and two time scales.

timescale process is shown in Fig. [3 The lower timescale is $\frac{1}{q}$, while the higher timescale is $\frac{1}{q^{2}}$.

During an On period of the higher timescale process, the lower timescale process alternates between $O n$ and $O f f$ periods, see Fig. 3. The number of times it alternates between the $O n$ and the Off periods is a geometric random variable with mean $q^{-1}$. Thus, there will be (on average) $\frac{1}{2 q}$ visits to the Off state of the lower timescale and $\frac{1}{2 q}$ visits to the $O n$ state of the lower timescale. Each visit to the On state of the lower timescale generates (on average) $\frac{1}{q}$ arrivals. Therefore, the probability that a given arrival occurs from leaving the off period of the higher timescale process is $\frac{1}{\frac{1}{2 q^{2}}+\frac{1}{2 q}+1}=\frac{(2 q)^{2}}{2+2 q+(2 q)^{2}}$. Similarly, the probabilities that a given arrival occurs for the $O f f$ and $O n$ periods of the lower scale process are $\frac{2 q}{2+2 q+(2 q)^{2}}$ and $\frac{2}{2+2 q+(2 q)^{2}}$, respectively. The inter-arrival time distribution for this two-scale point process is,

$$
f_{2 X}(x)= \begin{cases}\frac{2 \lambda e^{-\lambda x}+2 q \lambda q e^{-\lambda q x}+(2 q)^{2} \lambda q^{2} e^{-\lambda q^{2} x}}{2+2 q+(2 q)^{2}} & \text { for } x \geq 0 \\ 0 & \text { otherwise. }\end{cases}
$$

This point process consists of clusters, which in-turn are composed of clusters of arrivals. This behavior is shown in a realization of this point process over two timescales in Fig. 目

Now consider a point process constructed in a fashion as described above which contains $n$ timescales. This process may be viewed as the basic process embedded in the $O n$ state of the $n^{\text {th }}$ timescale. The time between visits to the $O n$ and $O f f$ periods in this $n$ timescale process is exponentially distributed with parameter $\lambda q^{n}$. The diagram for the $n$ timescale process is shown in Fig. [5. For each arrival generated from leaving an Off period at the hig hest timescale, (on average) $\frac{1}{2 q}$ arrivals will be generated from leaving an Off period at the next lower timescale, $\frac{1}{2 q^{2}}$ arrivals will be generated by the following Off period of the next timescale, etc. Continuing in this manner, for every event that occurs from leaving the Off period in the highest timescale, the average number of events that 
Table 1. Probabilities and rate of arrivals occurring on a particular timescale.

\begin{tabular}{|c|c|c|c|}
\hline \hline State & $\begin{array}{c}\text { Time } \\
\text { Scale }\end{array}$ & Probability & $\begin{array}{c}\text { Average } \\
\text { arrival rate }\end{array}$ \\
\hline off & $q^{-n}$ & $\frac{(2 q)^{n}}{2+\sum_{i=1}^{n}(2 q)^{i}}$ & $\lambda q^{n}$ \\
\hline$\vdots$ & $\vdots$ & $\vdots$ & $\vdots$ \\
\hline off & $q^{-j}$ & $\frac{(2 q)^{j}}{2+\sum_{i=1}^{n}(2 q)^{i}}$ & $\lambda q^{j}$ \\
\hline$\vdots$ & $\vdots$ & $\vdots$ & $\vdots$ \\
\hline off & $q^{-1}$ & $\frac{2 q}{2+\sum_{i=1}^{n}(2 q)^{i}}$ & $\lambda q$ \\
\hline on & $q^{-1}$ & $\frac{2+\sum_{i=1}^{n}(2 q)^{i}}{2+}$ & $\lambda$ \\
\hline \hline
\end{tabular}

occur due to the process being in the Off states of any timescale can be identified. Table 1 contai ns the probability of an arrival generated from a particular state.

Given that the process is in the Off period at timescale $i$, the time spent in this period is exponential with parameter $\lambda q^{i}$. Therefore, the inter-arrival time distribution of the overall point process is,

$$
f_{n} X(x)= \begin{cases}\frac{2 \lambda e^{-\lambda x}+\sum_{i=1}^{n}(2 q)^{i} \lambda q^{i} e^{-\lambda q^{i} x}}{2+\sum_{i=1}^{n}(2 q)^{i}} & \forall x \geq 0 \\ 0 & \text { otherwise. }\end{cases}
$$

It is easily verified that $\int_{0}^{\infty} f_{n} X(x)=1$. As the number of timescales approach infinity, interesting properties of the distribution of the time between clusters (inter-cluster gap) develop. Let $f_{X}(x) \equiv \lim _{n \rightarrow \infty} f_{n X}(x)$, which may be shown to be uniformly convergent (see Section [3.1).

Distribution of Inter-cluster Times A cluster at the $(i-1)$ timescale corresponds to an arrival at timescale $i$. If a cluster can be assumed to be a point (by compressing time by a factor of $q^{i-1}$ ) then the model may be assumed to start from scale $i$ and has $(n-i)$ timescales. Therefore, the inter-cluster times

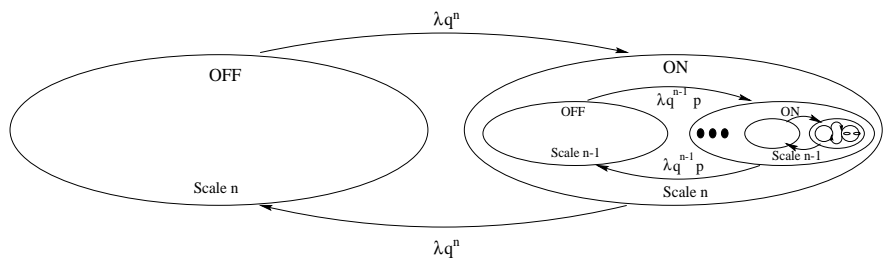

Fig. 5. Model to construct fractal point process over $n$ timescales. 
$Y$ have a probability density function,

$$
f_{n} Y(x)= \begin{cases}\frac{2 \lambda q^{i-1} e^{-\lambda q^{i-1} x}+\sum_{j=1}^{n-i+1}(2 q)^{j} \lambda q^{i+j-1} e^{-\lambda q^{i+j-1} x}}{2+\sum_{j=1}^{n-i+1}(2 q)^{j}} & \forall x \geq 0 \\ 0 & \text { otherwise. }\end{cases}
$$

Scaling $Y$ by $q^{i-1}$, the new random variable, $Y^{\prime}=q^{i-1} Y$, has a probability density function,

$$
f_{l} Y^{\prime}(x)= \begin{cases}\frac{2 \lambda e^{-\lambda x}+\sum_{j=1}^{l}(2 q)^{j} \lambda q^{j} e^{-\lambda q^{j} x}}{2+\sum_{j=1}^{l}(2 q)^{j}} & \forall x \geq 0 \\ 0 & \text { otherwise }\end{cases}
$$

where $l=n-i+1$. Let $f_{Y^{\prime}}(x) \equiv \lim _{l \rightarrow \infty} f_{l} Y_{Y^{\prime}}(x)$, which is also uniformly convergent.

Consider the complementary cumulative distribution function (ccdf) for $X$ and $Y$. It is seen that

$$
P(X>u)=\int_{u}^{\infty} f_{X}(x) d x=\int_{u}^{\infty} f_{Y^{\prime}}(x) d x=P\left(Y^{\prime}>u\right) .
$$

(Note that these are the same values of $u$, but on different timescales). Therefore, the probability that an inter-cluster time is greater than $u$ of its time units is equal to the probability that the inter-arrival time of points is greater than $u$ of its time units. Since, $n \rightarrow \infty(l \rightarrow \infty)$, this result holds for all finite timescales. Thus, a burst on any timescale will be statistically the same, a fundamental characteristic of a fractal point process.

\section{Analysis of the Model}

The model delveloped had no strict restrictions on the parameters used to describe it. The only conditions on the parameters were $\lambda>0$ and $0<q<1$. The density function has to be investigated further to find whether any more restrictions on the model parameters are needed to make the probability density function a valid density function, as $n \rightarrow \infty$.

\subsection{Uniform Convergence of the Probability Density Function}

Using the model described in Section 2, points (arrivals) can be generated with an inter-arrival distribution given in (2). Several characteristics are observed in this process as the number of timescales embeddings approach infinity, namely, the inter-cluster time distributions were statistically the same and only differed in timescale. Convergence of this distribution $f_{X}(x)$ for any timescale is studied in this section. Uniform convergence of $f_{X}(x)=\lim _{n \rightarrow \infty} f_{n X}(x)$ can be shown by applying the Weierstrass' M-test [16], as follows. For $x<0$, the probability density function is always zero. For $x \geq 0$, the probability density function can 
be analyzed by considering it to be the quotient of two series. The probability density function $f_{X}(x)$, for $x \geq 0$, is,

$$
f_{X}(x)=\frac{2 \lambda e^{-\lambda x}+\sum_{i=1}^{\infty}(2 q)^{i} \lambda q^{i} e^{-\lambda q^{i} x}}{2+\sum_{i=1}^{\infty}(2 q)^{i}} .
$$

When $q \in(0,0.5)$, the denominator in the above expression evaluates to $\frac{2-2 q}{1-2 q}$. The infinite series in the numerator is $\lambda\left[2 e^{-\lambda x}+\sum_{i=1}^{\infty}\left(2 q^{2}\right)^{i} e^{-\lambda q^{i} x}\right]$. Let $M_{n}=$ $\left(2 q^{2}\right)^{n}$ for $n>0$ and $M_{n}=2$ for $n=0$. Then, $\sum_{n=0}^{\infty} M_{n}$ converges absolutely if $2 q^{2}<1$. For $q \in(0,0.5), 2 q^{2} \leq 1$ and $\sum_{n=0}^{\infty} M_{n}$ converges to $\frac{2-2 q^{2}}{1-2 q^{2}}$. If $g_{n}(x)$ is defined as

$$
g_{n}(x)=\left\{\begin{array}{ll}
2 e^{-\lambda x} & \text { for } n=0 \\
\left(2 q^{2}\right)^{n} e^{-\lambda q^{n} x} & \text { for } n>0
\end{array},\right.
$$

then it is seen that for all $x>0$ and $\lambda>0,\left|g_{n}(x)\right| \leq M_{n}, \forall n>0$ and therefore, $N r\left\{f_{X}(x)\right\}$ converges uniformly $\forall x \geq 0$. Thus, it is seen that $f_{X}(x)$ converges uniformly $\forall x \geq 0$, when the generation process consists of infinite number of timescale embeddings.

\subsection{Moments of the Density Function}

The mean inter-arrival time for this process with $0 \leq q \leq 0.5$ may be evaluated as,

$$
\begin{aligned}
E[x] & =\int_{0}^{\infty} \frac{2 \lambda x e^{-\lambda x}+\sum_{i=1}^{n}(2 q)^{i} \lambda q^{i} x e^{-\lambda q^{i} x}}{2+\sum_{i=1}^{n}(2 q)^{i}} d x \\
& =\frac{\frac{1}{\lambda}\left(2+\sum_{i=1}^{n} 2^{i}\right)}{2+\sum_{i=1}^{n}(2 q)^{i}} .
\end{aligned}
$$

As $n \rightarrow \infty$,

$$
E[X]=\frac{\frac{1}{\lambda}\left(2+\sum_{i=1}^{\infty} 2^{i}\right)}{2+\sum_{i=1}^{\infty}(2 q)^{i}}
$$

Therefore, as the number of timescales embedded in the process approaches infinity, the mean inter-arrival time approaches infinity and the mean arrival rate $E[X]^{-1}$ approaches zero. This result is another fundamental and necessary characteristic of a self-similar point process [1].

\subsection{Behavior of the Density Function Compared to a Pareto Distribution}

A point process can be called fractal if the following phenomenon is observed. On a particular timescale, the point process looks bursty, with a lot of arrivals 
clustered around one point. If the time axis is dilated about this point and the cluster is closely observed, the cluster looks bursty again leading to the formation of clusters over the new timescale. If on continuous dilations of the timescale about a point where the arrivals appear to be clustered, results in clustering of points again over several timescales, the point process is said to exhibit fractal properties. Alternatively, it can be explained as follows. The distribution of the inter-arrival time on a particular timescale and the distribution of the interarrival time on another timescale, differ only in scale. In [1, it is shown that the only distribution that satisfies the above condition, i s the Pareto distribution. A Pareto distribution can be described by its probability density function,

$$
f_{X}(x)= \begin{cases}K_{\epsilon} x^{-(\gamma+1)} & x \geq \epsilon \\ 0 & \text { otherwise }\end{cases}
$$

where $K_{\epsilon}$ is a normalizing constant to make $f_{X}(x)$ a valid density function.

A Pareto distribution may be expressed as a weighted sum of exponentials by considering the Gamma function. The Gamma function is, $\Gamma(D)=$ $\int_{0}^{\infty} e^{-y} y^{D-1} d y$. Allowing $y=b x, d y=x d b$ and observing that the limits remain the same, the following equation is obtained, $x^{-D}=\int_{0}^{\infty} e^{-b x} b^{D-1} d b \Gamma(D)^{-1}$. Approximating this integral by a summation, $x^{-D}$ may be written as a sum of weighted exponentials. This approach may be used to relate the fractal pa rameter $D$, to the parameters of this model. If the probability density function of the inter-arrival times for a point process generated by this model behaves as $x^{-D}$, a relationship between the model parameters and the fractal $D$ may be obtained. Approximating the integral,

$$
x^{-D}=\Gamma(D)^{-1} \sum_{m=0}^{\infty} e^{-b_{m} x} b_{m}^{D-1}\left(b_{m}-b_{m+1}\right) .
$$

Using the substitution $b_{m}=\lambda q^{m}$, the above equation becomes,

$$
\begin{aligned}
x^{-D} & =\Gamma(D)^{-1} \sum_{m=0}^{\infty} e^{-\lambda q^{m} x}\left(\lambda q^{m}\right)^{D-1}\left(\lambda q^{m}\right)(1-q) \\
& =G(D) \sum_{m=0}^{\infty} \lambda\left(q^{m}\right)^{D} e^{-\lambda q^{m} x},
\end{aligned}
$$

where $G(D)=\Gamma(D)^{-1} \lambda^{D-1}(1-q)$.

The probability density function can be written as,

$$
f_{X}(x)=\frac{2-2 q}{1-2 q}\left[\lambda e^{-\lambda x}+\sum_{m=0}^{\infty} \lambda\left(2 q^{2}\right)^{m} e^{-\lambda q^{m} x}\right] .
$$

For large values of $x$ the contribution of the initial terms in (7) and (8) are negligible. Comparing the remaining terms of (77) to those of (87), it is seen that $q^{m D}=\left(2 q^{2}\right)^{m}$ or

$$
D=\frac{\log 2}{\log q}+2
$$



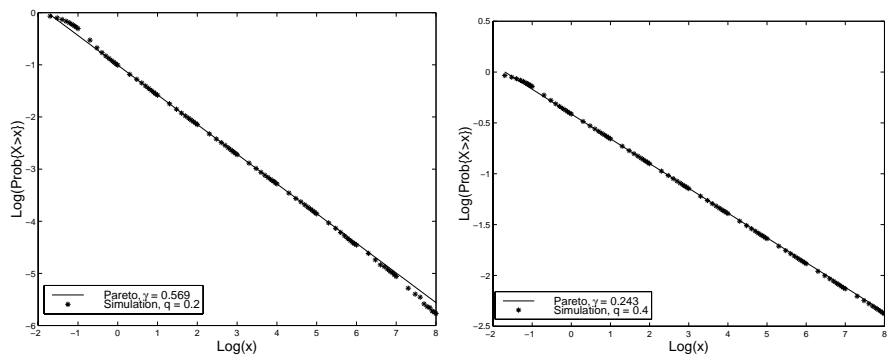

Fig. 6. Loglog plot of ccdf of the simulated data vs. Pareto distribution.

and $\gamma=(D-1)$, where $\gamma$ is the fractal exponent of the Pareto distribution in (6). It is seen that for $0<q<0.5$, then $0<\gamma<1$, which is the range for which the point process in [1] behaves as a fractal point process.

\section{Results}

In this section, the simulations using the above described model are compared to the theoretical Paretian distributions. Fig. 6] compares the complementary cumulative distribution function of the point process simulated to that of the corresponding Pareto distribution. It may be seen from these plots that the probability density function of the point process generated by this model approaches a Paretian distribution. Fig. [7 shows the counting process of a single realization over three different time scales. The first plot contains the first 10000 points of the simulation. The plot shows the strong clustering of data. On zooming in, on the first cluster, the second plot is obtained, which again shows strong clustering of data. On further zooming in, the third plot is obtained, which shows strong clustering of points again. The clustering of clusters and the reduction of a cluster into a point on higher timescales may be illustrated in this figure.

\section{Conclusion}

This model was developed based on the fact of clustering of arrivals within clusters as in present day telecommunication traffic. The concept of embedding an $O n$-Off process into another $O n$-Off process gives more structure to the understanding of these clusters. In [1], it is shown that if the inter-arrival times follow a Paretian distribution, the inter-cluster time distribution differ from the interarrival time distribution only in scale. The analysis of the model developed, shows that the distribution of the inter-cluster time and that of the inter-arrival time of the point process, differ only in scale. In addition, the model allows for efficient synthesis of the process. The simulation results show that the complementary cumulative distribution function of the inter-arrival times behaves as a Pareto distribution. 

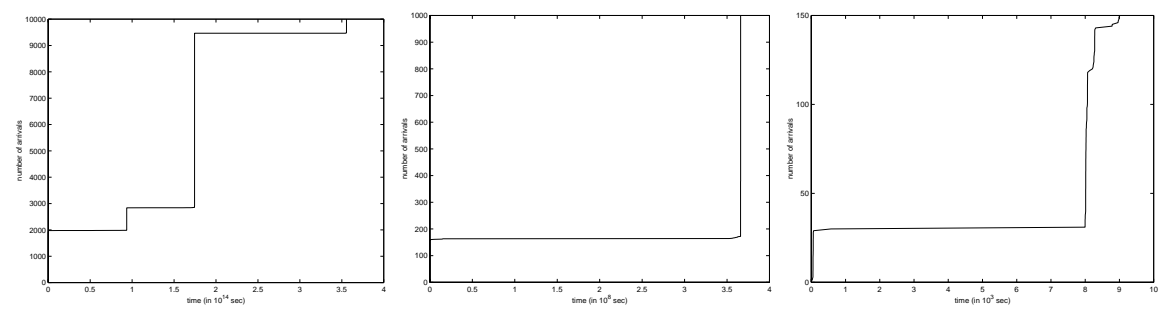

Fig. 7. Counting process showing strong clustering of arrivals over different timescales.

\section{A Geometric Sum of Exponential Random Variables}

Theorem 1. If $S=\sum_{i=1}^{N} X_{i}$, where $X_{i}$ s are independent identically distributed exponential random variables with parameter $\lambda$ and $N$ is a geometric random variable with parameter $q$, then $S$ is an exponential random variable with parameter $\lambda q$.

Proof:

The distribution of $S$ is found by first conditioning on $N=n$,

$$
f_{S \mid N=n}(s)= \begin{cases}\frac{\lambda^{n} s^{n-1}}{(n-1) !} e^{-\lambda s} & \forall s \geq 0 \\ 0 & \text { otherwise. }\end{cases}
$$

Taking expectation with respect to $N$,

$$
\begin{aligned}
f_{S}(s) & =\sum_{n=1}^{\infty} \frac{\lambda^{n} s^{n-1} p^{n-1} q e^{-\lambda s}}{(n-1) !} \\
& =\lambda q e^{-\lambda s} e^{-\lambda s p} \\
& =\lambda q e^{-\lambda s q} .
\end{aligned}
$$

Thus, the probability density function of $S$ is,

$$
f_{S}(s)= \begin{cases}\lambda q e^{-\lambda q s} & \forall s \geq 0 \\ 0 & \text { otherwise. }\end{cases}
$$

\section{B Algorithm for Generating a Self-Similar Point Process}

The algorithm used to synthesize the fractal point process of Section 2 , is described in this section. This algorithm assumes embedding of $O n$-Off processes over infinite timescales. The other parameters to be specified are $\lambda$ and $\gamma . \lambda$ is determined by the value of time above which the probability density function or the complementary cumulative distribution function behaves as a Pareto distribution. This may be viewed as setting the value of $\epsilon$ in (??). $\gamma$ is the parameter of the Pareto distribution. 
This algorithm consists of four functions. The variables currentState and $q$ are used in the algorithm. currentState corresponds to the state and the timescale in which the generation process is residing. currentState $=1$ corresponds to the $O n$ state of the lowest timescale. currentState $=2$ corresponds to the Off state of the lowest timescale. Continuing similarly currentState $=n$ corresponds to the Off state of the $(n-1)^{t h}$ timescale. The value of $q$ depends on the expone nt of the Pareto distribution, to which the probability density function of the inter arrival time approaches. It is calculated from the value of $\gamma$, using relation (9). A random initial state is chosen according to the probabilities given in Table 1 The process starts off initially with currentState set to this number. The second function NEXTSTATE computes the state to which the generation process will jump next. This function uses two other functions SUCCESS and FA ILURE. SUCCESS and FAILURE describe the sequence of events that occur when the process has to leave any particular state.

\section{$\operatorname{GENERATE}(\lambda, \gamma)$}

1. $q \leftarrow 2^{-\frac{1}{1-\gamma}}$

2. initialize currentState

3. time $\leftarrow 0$

4. while (1)

5. do generate exponential random number, $\exp$, with parameter $\lambda q^{\text {currentState }-1}$

6. $\quad$ time $\leftarrow$ time + exp

7. Generate an arrival at time seconds

8. currentState $\leftarrow$ NEXTSTATE(currentState)

\section{NEXTSTATE(currentState)}

1. Generate a uniform random number unirand $\in(0,1)$.

2. if (unirand $>q$ )

3. $\quad$ currentState $\leftarrow$ SUCCESS(currentState)

4. else

5. currentState $\leftarrow$ FAILURE (currentState)

6. return currentState

\section{SUCCESS(currentState)}

1. if (currentState $=1$ )

2. return currentState

3. else

4. $\quad$ currentState $\leftarrow$ currentState -1

5. Generate uniform random number unirand $\in(0,1)$

6. if (unirand $<0.5)$

7. return currentState

8. else

9. return SUCCESS(currentState)

FAILURE(currentState) 
1. currentState $\leftarrow$ currentState +1

2. Generate uniform random number unirand $\in(0,1)$

3. if (unirand $>q)$

4. return currentState

5. else

6. return FAILURE(currentState)

\section{References}

1. B. B. Mandelbrot, "Self-similar error clusters in communication systems and the concept of conditional stationarity," IEEE Transactions on Communication Technology, vol. COM-13, pp. 71-90, March 1965.

2. J. Beran, Statistics for Long-Memory Process. New York: Chapman and Hall, 1994.

3. M. E. Crovella and M. S. Taqqu, "Estimating the heavy tail index from scaling properties," Methodology and Computing in Applied Probability, vol. 1, no. 1, 1999 to appear.

4. S. B. Lowen and M. C. Teich, "Fractal renewal processes generate $1 / \mathrm{f}$ noise," Physics Review, vol. E, no. 47, pp. 992-1001, 1993.

5. B. K. Ryu and S. B. Lowen, "Point Process Approaches to the Modeling and Analysis of Self-Similar Traffic - Part I: Model Construction," in Proc. IEEE INFOCOM'96, pp. 1468-1475, March 1996.

6. B. Tsybakov and N. D. Georganas, "Self-similar processes in Communications Networks," IEEE Transactions on Information Theory, vol. 44, pp. 1713-1725, September 1998.

7. W. Leland, M. Taqqu, W. Willinger, and D. Wilson, "On the self-similar nature of Ethernet traffic.," IEEE/ACM transactions on Networking, vol. 2, pp. 1-15, February 1994.

8. V. Paxson and S. Floyd, "Wide-area traffic: the failure of Poisson Modeling," in Proc. Sigcomm'94, pp. 257-268, October 1994.

9. M. Parulekar and A. Makowski, "Tail Probabilities for a multiplexer with selfsimilar traffic," in Proc. IEEE INFOCOM'96, pp. 1452-1459, March 1996.

10. A. T. Andersen and B. F. Nielsen, "An application of superposition of two state Markovian sources to the modelling of self-similar behavior," in Proc. IEEE INFOCOM'97, 1997.

11. A. Feldmann and W. Whitt, "Fitting Mixture of Exponentials to Long-Tail Distributions to Analyze Network Performance Models," Performance Evaluation, vol. 31, pp. 245-279, January 1998.

12. N. Likhanov, B. Tsybakov, and N. D. Georganas, "Analysis of an ATM buffer with self-similar ("fractal") input traffic," in Proc. IEEE INFOCOM'95, 1995.

13. P. R. Jelenkovic and A. A. Lazar, "Asymptotic Results for Multiplexing Subexponential On-Off Processes," Advances in Applied Probability, vol. 31, no. 2, 1999 to appear.

14. A. Erramilli, R. P. Singh, and P. Pruthi, "Modeling Packet Traffic with Chaotic Maps," Royal Institute of Technology, ISRN, KTH/IT/R-94/18, August 1994.

15. W. M. Lam and G. W. Wornell, "Multiscale Representation and Estimation of Fractal Point Processes," IEEE Transactions on Signal Processing, vol. 43, pp. 2606-2617, November 1995.

16. W. Rudin, Priniciples of Mathematical Analysis. McGraw Hill, 1976. 